\title{
Research on the Physiological and Psychological Ability Indexes of Coal Tunneling Workers
}

\author{
Lu Cao $^{1 *}$, Hanqing Chen ${ }^{2}$ \\ ${ }^{1}$ School of Economics and Management, Anhui University of Science and Technology, Huainan 232001, China \\ ${ }^{2}$ School of Civil Engineering and Architecture, Anhui University of Science and Technology, Huainan 232001, China \\ *Corresponding author: Lu Cao, caoluazj@139.com
}

\begin{abstract}
Studies have shown that accidents caused by human error account for $70-80 \%$ of the total number of accidents, and are the primary cause of casualties. In order to reduce the risk of safety accidents and reduce the human-caused accident rate, the evaluation index of safety occupational adaptability of coal excavation workers has been studied. Questionnaire survey method was used to conduct 13 physical and psychological abilities tests on 50 coal tunneling workers, and select the core ability indicators that reflect the mental load of the workers and the individual priority allocation of psychological resources. Secondly, using the Delphi method and the quartile method, the opinions of 20 experts in related fields were sought anonymously, and the main physical and mental ability indicators were screened and filtered through scoring methods. Finally, six indicators of "accuracy, attention, rapid response ability, proficiency, judgment, and foresight" were determined as the main physical and psychological indicators of coal tunneling workers. This study fills up the gap of safety occupational adaptability in the field of mining construction, and has important theoretical value and practical significance.
\end{abstract}

Keywords: Physical and mental abilities; Psychological resources; Mental load; Safety occupational adaptability

Publication date: August 2021; Online publication: August 30, 2021

\section{Introduction}

In recent years, the number of deaths from accidents in China has dropped from a historical peak of 139,000 in 2002 to 29,000 in 2019. The number of production safety accidents and deaths has declined for 17 consecutive years, but the volume of accidents is still very large, as shown in Figure 1. At the same time, accidents related to production have also shown a trend of increase from traditional high-risk industries to other industries, and the safety in production is still severe and complicated ${ }^{[1]}$.

Studies have found that accidents caused by human error accounted for $70-80 \%$ of the total number of accidents ${ }^{[2]}$, which is the primary cause of casualties. Therefore, studying the safety and adaptability relationship between humans and machines is essential to ensure system efficiency and prevent and control human accidents ${ }^{[3]}$.

Since the 1960s, foreign scholars have begun to study the safety and occupational adaptability of drivers, welders, electricians, cranes, furnace workers and other special operators ${ }^{[4]}$. Among them, the American Hester company produced a set of occupational adaptability evaluation system called MVE, which divides the individual evaluation indicators in detail (including 7 in environmental adaptability, 9 in psychological limits, 9 in interpersonal relationships; a total of 25 indicators) to assess the suitability of 2,000 occupations. Due to its good operability and portability, the system was quickly applied in Canada, Australia, Japan, and other countries ${ }^{[5]}$.

However, China is relatively new to the research on safety occupational adaptability, and domestic scholars did not start researching in this field until the 1980s. Jin et al. of the Anhui Sanlian Accident 
Prevention Research Institute first conducted a study on the safety adaptability of drivers, constructed a three-dimensional model of "characteristic-environment-one-period" of accident propensity, and compiled the driver's safety status measurement table; this model has become a must-test item for drivers before they take up their jobs ${ }^{[6]}$. Secondly, Song ${ }^{[7]}$ and Ye et al. ${ }^{[8]}$ studied the occupational adaptability of electric operators and train drivers, and achieved good results. In addition, Li and Song carried out safety occupational adaptability research for power system operators, and proposed six test indicators such as aiming accuracy, average speed prediction time, attention, speed prediction variation coefficient, complex response variation coefficient, and low-speed concave face motion tracking time as a comprehensive evaluation index for their occupational adaptability ${ }^{[9]}$.
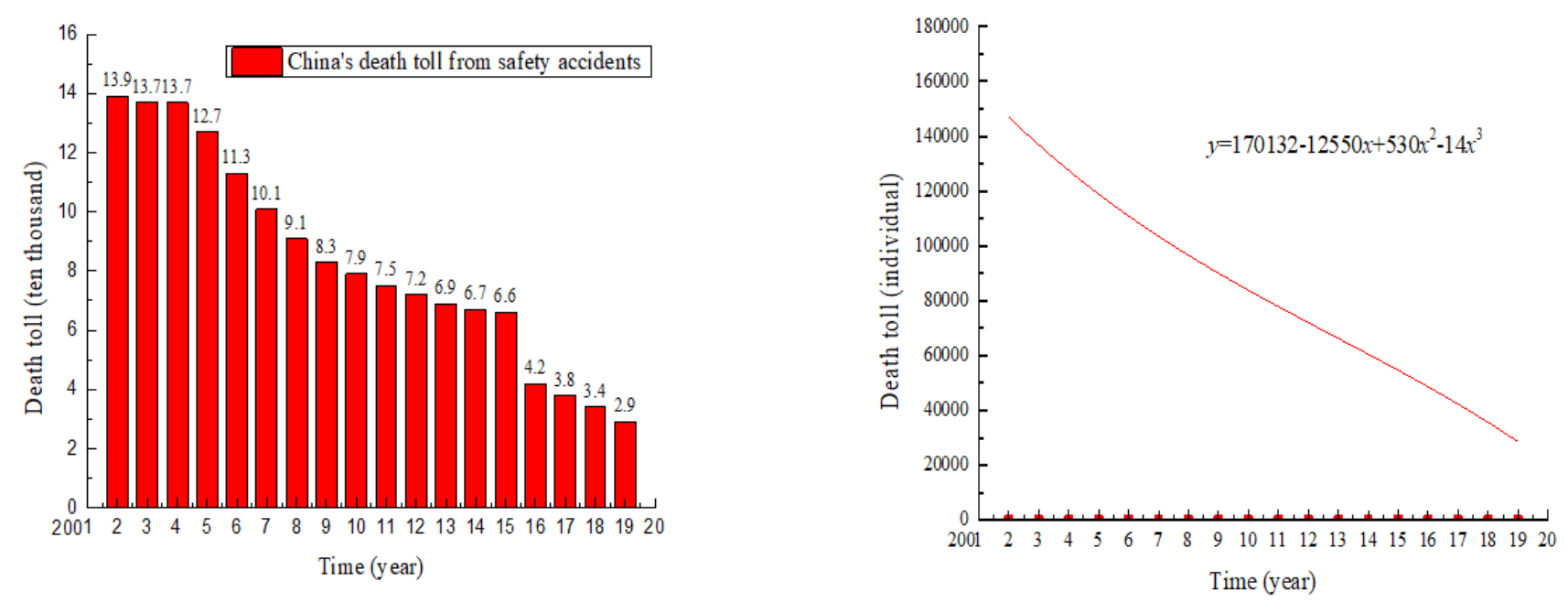

Figure 1. Statistics on the number of deaths from accidents in China over the years

Safety occupational adaptability evaluation is a new subject and method for measuring and evaluating people's physical and psychological comprehensive attributes. It can be used for accident prevention and personnel selection ${ }^{[10]}$. Through the safety quality ability assessment of operators, the human body's ability to protect itself from machines, materials, objects, and the environment can be explored; it does not only improve the safety quality of workers, but also the ability to use and allocate safe psychological resources [11]. However, there are few studies on the safety occupation adaptability for coal tunneling workers at present ${ }^{[4]}$. Therefore, the research on the physiological and psychological ability indexes of safety occupation adaptability has important theoretical value and practical significance.

The long-term exposure to high temperature, high humidity, noise and vibration operating environment of coal tunneling workers have important impact on the miners' psychological load. In order to reduce the rate of safety production accidents caused by operators' errors, the research on physiological and psychological ability indexes of safety occupation adaptability was carried out for a tunneling team in Guqiao Mine, Huainan. In this study, 13 physical and psychological indicators were selected through questionnaire surveys. Then, the Delphi method was used to consult the expert opinions anonymously, and then the quartile method was used to process the expert opinions, and to identify the indicators and weights ${ }^{[12]}$. Based on this, the main physical and mental ability indicators are finally selected.

\section{Main indicators selected by the questionnaire survey}

To carry out safety occupation adaptability research for special operators, the key is to analyze the physiological and psychological ability indicators ${ }^{[4]}$. Based on this, domestic scholars have carried out a lot of research on physical and mental ability indicators. Wu et al. believe that the operators of dangerous 
devices should have strong physical and psychological qualities, and thus proposed 27 physical and psychological ability indicators ${ }^{[12]}$. Ye et al. believe that the eyesight of railway drivers is often in a state of high tension and need to deal with various emergency responses (so, they were selected based on very strict career criteria), and proposed two physiological indicators and seven psychological indicators ${ }^{[13]}$. Jin et al. carried out a study on motor vehicle drivers and gave six physical and psychological indicators such as speed estimation, operating function, complex reflection judgment, deep vision, night vision, and dynamic vision ${ }^{[14]}$. Ou et al. believe that the operators of electric power enterprises work in the environment of noise, vibration, and electromagnetic radiation for a long time, and they also need to shift work to complete their work tasks, thus putting forward six physical and psychological indicators such as attention distribution and shifting, complex response, speed prediction, accuracy, eye-hand coordination, and job stability ${ }^{[15]}$. Based on this, Li and Song selected six out of 13 physical and mental ability indicators as the main indicators for power operator, such as accuracy, attention, rapid response ability, proficiency, judgment, and foresight ${ }^{[9]}$.

In summary, it can be seen that for operators of different special occupations, their physical and mental ability indicators are universal. Attention, speed prediction, complex reaction ability, eye-hand coordination, etc. are some of the examples. In view of the fact that coal tunneling operators and power operation operators are relatively similar in work characteristics, psychological load and safety adaptability requirements. Therefore, based on $\mathrm{Li}$ and Song's research methods on electric power operators ${ }^{[9]}$, the physiological and psychological factors of coal tunneling operators were screened.

In order to select the main physical and psychological abilities, a study was carried out on 50 workers from an tunneling team in Guqiao Mine, Huainan. Through the questionnaire survey, each of the test subjects was assessed based on the 13 factors, namely "accuracy, stability, continuity, willpower, attention, memory, quick response ability, coordination, proficiency, judgment, decision-making ability, understanding, and foresight," and the importance of the factors was sorted accordingly. The degree of importance is divided into five levels: important, more important, general, less important, and unimportant. Based on this, the "scale table" was used to score the above indicators according to their importance by using a 1 to 5 point system; a rank table was compiled. Through the collection, sorting and statistical analysis of the data filled by the operators, the survey results are shown in Table 1.

It can be seen from Table 1 that for coal tunneling workers, the physical and psychological indicators are ranked according to the total score of the options: attention $(221)>$ accuracy (198) $>$ judgment (183) > quick response ability $(155)>$ proficiency $(118)>$ foresight $(114)>$ stability $(80)>$ comprehension $(74)>$ coordination (73) $>$ decision-making ability (66) $>$ memory $(60)>$ coherence $(28)>$ willpower (18). Based on this, six physical and psychological abilities, including quasi-attention, accuracy, judgment, rapid response ability, proficiency, and predictability, can be screened out as the main research indicators according to the score.

\section{Main indicators selected by the Delphi method and the quartile method}

In addition, through the Delphi method and the quartile method, the main indicators of the physical and psychological capabilities of coal mine excavators can also be determined. The specific implementation methods are as follows:

Firstly, the Delphi method was used to anonymously consult 20 experts in related fields, including five technical persons in charge of mine construction of Huainan Mining Group, five experts in safety management departments, five senior engineers of design units, five professors of Anhui University of Science and Technology, to determine the importance of each quality index by scoring 13 physical and psychological indicators of coal mine tunneling workers.

Secondly, in order to improve the concentration of data, the quartile method is used to process the 
expert score sheet, and the lower quartile and upper quartile of 13 physical and mental ability indicators were determined by sorting their score from low to high. For indicators with a concentration of less than or equal to 1, only one round of consultation will be conducted; for indicators with a concentration of greater than 1, a second round of consultation is required. Finally, the scores of 13 physical and psychological ability indicators of coal mine excavation workers were determined.

Table 1. Survey score statistics of coal mining employees' safety quality and ability

\begin{tabular}{lcl}
\hline \multicolumn{1}{c}{ Ability options } & Option total score (minutes) & \multicolumn{1}{c}{ Reorder by score (minutes) } \\
\hline 1 Accuracy & 198 & 5 Attention (221) \\
2 Stability & 80 & 1 Accuracy (198) \\
3 Continuity & 28 & 10 Judgment (183) \\
4 Willpower & 18 & 7 Quick response ability (155) \\
5 Attention & 221 & 9 Proficiency (118) \\
6 Memory & 60 & 13 Predictability (114) \\
7 Quick response ability & 155 & 2 Stability (80) \\
8 Coordination & 73 & 12 Understanding (74) \\
9 Proficiency & 118 & 8 Coordination (73) \\
10 Judgment & 183 & 11 Decision-making capacity (66) \\
11 Decision-making capacity & 66 & 6 Memory (60) \\
12 Understanding & 74 & 3 Continuity (28) \\
13 Predictability & 114 & 4 Willpower (18) \\
\hline
\end{tabular}

Table 2. Index score summary and ranking table

\begin{tabular}{lll}
\hline \multicolumn{1}{c}{ Queue number } & Total score (minutes) & \multicolumn{1}{c}{$\begin{array}{c}\text { Reorder by score (expert consultation } \\
\text { method; point) }\end{array}$} \\
\hline 1 Accuracy & 92 & 5 Attention (93) \\
2 Stability & 53 & 1 Accuracy (92) \\
3 Continuity & 32 & 7 Quick response ability (91) \\
4 Willpower & 33 & 10 Judgment (90) \\
5 Attention & 93 & 9 Proficiency (70) \\
6 Memory & 35 & 13 Predictability (67) \\
7 Quick response ability & 91 & 12 Understanding (55) \\
8 Coordination & 52 & 11 Decision-making capacity (54) \\
9 Proficiency & 70 & 2 Stability (53) \\
10 Judgment & 90 & 8 Coordination (52) \\
11 Decision-making capacity & 54 & 6 Memory (35) \\
12 Understanding & 55 & 4 Willpower (33) \\
13 Predictability & 67 & 3 Continuity (32) \\
\hline
\end{tabular}


Through two rounds of survey results, the total scores of 13 physical and psychological indicators of coal mining operators were processed, and they were ranked according to importance. See Table 2 for details.

It can be seen from Table 2 that through two rounds of expert consultation with the Delphi method and the quartile method, the physical and psychological indicators of coal mining operators can be sorted by the total score of the options: attention (93) > accuracy (92) > quick response ability (91) > judgment (90) $>$ proficiency $(70)>$ foresight $(69)>$ understanding (55) > decision-making ability (54) > stability (53) $>$ coordination (52) > memory (35) > willpower (33) > coherence (32). Based on this, six physical and psychological abilities, i.e., attention, accuracy, rapid response ability, judgment, proficiency, and predictability, were identified as research indicators according to the score.

In summary, whether it is the questionnaire survey method for the operators, or the Delphi method and the quartile method for related experts, six physical and psychological indicators can be screened out, which include accuracy, attention, rapid response ability, proficiency, judgment, and predictability. These indicators do not only reflect the mental load of the workers, but also reflect the individual's core ability to prioritize the allocation of psychological resources.

\section{Conclusion}

Effective physical and psychological index research is the key to evaluating the physical and psychological abilities of coal mine excavators. A questionnaire survey was carried out on coal mine tunneling workers, and 13 physical and mental ability indicators were scored according to the "scale table," and the total score of each indicator was calculated. Based on this, the various indicators are sorted according to the total score: attention $(221)>$ accuracy $(198)>$ judgment $(183)>$ quick response ability $(155)>$ proficiency $(118)>$ foresight $(114)>$ stability $(80)>$ comprehension $(74)>$ coordination $(73)>$ decision-making ability (66) $>$ memory (60) > coherence (28) > willpower (18). Therefore, six physical and psychological abilities, i.e., quasi-attention, accuracy, judgment, rapid response ability, proficiency, and predictability, can be screened out as research indicators for safety occupational adaptability according to the score.

Through anonymous consultation with experts in related fields, the main indicators of the physical and psychological capabilities of coal mine excavators can also be determined. First, the Delphi method was used to anonymously consult 20 experts in related fields, and scored 13 physical and mental ability indicators. Then, the quartile method was used to process the expert score sheet to determine the total scores of 13 physical and mental abilities of coal miners. Finally, the physical and psychological indicators of coal miners were sorted according to the total score: attention (93) > accuracy (92) > speed-determining ability (91) $>$ judgment $(90)>$ proficiency $(70)>$ foresight (69) $>$ comprehension (55) > decision-making ability (54) $>$ stability (53) > coordination (52) > memory (35) > willpower (33) > coherence (32). Therefore, six physical and psychological abilities, including attention, accuracy, rapid response ability, judgment, proficiency, and predictability, can be screened out as research indicators according to the score.

\section{Disclosure statement}

The authors declare that there is no conflict of interest.

\section{References}

[1] Duan L-Z, 2006, Study on the Safety Management in Construction. Chongqing University, Chongqing.

[2] Wen D-D, 1996, On Human Error. China Safety Science Journal, 1996(6), Supp.: 85-9.

[3] Li S, Su L-B, Song S-X, 2010, Review of Studies on Psychological Workload of Workers in ManMachine System. Journal of Beijing Jiaotong University (Social Sciences Edition), 9(3): 54-8. 
[4] Li S, 2010, The Study of the Safety Occupation Aptitude Based on Mental Resource. Beijing Jiaotong University, Beijing.

[5] Liu C-Q, Xu G-G, Shi M, 2001, The Revision of Hearst's Career Assessment System and the Initial Establishment of Regional Norms. New Exploration of Psychology, 20(80): 51-65.

[6] Jin H-Q, 1996, Research and Application of Occupational Suitability of Chinese Motor Vehicle Drivers. Chinese Journal of Trauma, 1(32): 171-2.

[7] Song S-X, 2003, Accident Tendency and Human Factor Management. China Power Enterprise Management, 2003(12): 32-3.

[8] Ye L, Xiang F-F, Shen M, et al., 2003, Study on Human Safety Evaluation. China Safety Science Journal, 13(10): 11-4.

[9] Li S, Song S-X, 2010, Research on Safety Occupational Adaptability Indexes of Power System Operators. Productivity Research, 7(36): 97-9.

[10] Gao C-S, Zhu Z-S, Lu D-M, 1996, Evaluation and Application of Occupational Adaptability Test. Engineering Safety and Dust Prevention, 1996(1): 21-5.

[11] Jin H-Q, 2000, Test and Evaluation of Occupational Suitability Inspection System for Electrical Workers. Ergonomics, 2000(2): 54-5.

[12] Wu K, Zhang B-D, Yao H-X, 1997, Strategy and Method for Studying the Professional Adaptability for Operators of Dangerous Installation. China Safety Science Journal, 7(4): 17-22.

[13] Ye L, Shen M, Fu J-F, et al., 1997, Study on the Relationship between Engine Drivers' Physiological and Psychological Diatheses and Safe Driving on Railway. China Safety Science Journal, 7(6): 53 -7.

[14] Jin H-Q, Zhang S-L, Dai P, et al., 2000, Research on Comprehensive Evaluation of Motor Vehicle Drivers' Psychological Quality. Chinese Journal of Epidemiology, 21(5): 369 -71.

[15] Ou S-C, Ye L, Shen M, et al., 2003, Research on Occupational Adaptability of Power Enterprise Operators. Northern Economy, 2003(10): 39-43. 